IJMMS 26:8 (2001) 497-511

PII. S0161171201010407

http://ijmms.hindawi.com

(c) Hindawi Publishing Corp.

\title{
THE METHOD OF AVERAGING AND FUNCTIONAL DIFFERENTIAL EQUATIONS WITH DELAY
}

\author{
MUSTAPHA LAKRIB
}

(Received 2 October 2000)

\begin{abstract}
We present a natural extension of the method of averaging to fast oscillating functional differential equations with delay. Unlike the usual approach where the analysis is kept in an infinite-dimensional Banach space, our analysis is achieved in $\mathbb{R}^{n}$. Our results are formulated in classical mathematics. They are proved within Internal Set Theory which is an axiomatic description of nonstandard analysis.
\end{abstract}

2000 Mathematics Subject Classification. 34C29, 34K25, 34K20, 03 H05.

1. Introduction. An important tool in the rigorous study of differential equations with a small parameter is the method of averaging, which is well known for ordinary differential equations $[1,9,13,14]$ and for functional differential equations with small delay $[6,7,17]$. In both cases, the corresponding averaged equations are ordinary differential equations. However, for fast oscillating functional differential equations with large bounded delay, the method of averaging is not nearly so developed as in the two previous cases. Among recent works devoted to this last case, we will cite the paper of Hale and Verduyn Lunel [8]. Without going into details, we will emphasize, in this work, that the authors introduce an extension of the method of averaging to abstract evolutionary equations in Banach spaces. In particular, they rewrite a functional differential equation with delay as an ordinary differential equation in an infinite dimensional Banach space and proceed formally from there.

In this paper, we develop an improved theory of averaging for functional differential equations with delay under smoothness hypotheses that are less restrictive than those of [8]. Also all our analysis is kept in $\mathbb{R}^{n}$. This is performed in Section 2. There, we state closeness of solutions of the averaged and original equations on finite time intervals (Theorem 2.1). We also investigate the long time behaviour of the solutions of the original equation (Theorem 2.5). The proofs of Theorems 2.1 and 2.5 are established within an axiomatic description of Robinson's Nonstandard Analysis (NSA) [12], namely Internal Set Theory (IST), proposed by Nelson [11]. Section 3 starts with a short tutorial on IST. Then we present the nonstandard translates (Theorems 3.5 and 3.7) in the language of IST of Theorems 2.1 and 2.5. We end this section with an external characterization of the uniform asymptotic stability which is the main assumption for the validity of the result of Theorem 3.7. Finally, in Section 4 we give the proofs of Theorems 3.5 and 3.7. As ordinary differential equations and functional differential equations with small delay are special cases of functional differential equations with delay, the proofs developed in this section provide alternative 
proofs to the techniques of averaging on these equations found, for example, in $[13,14]$.

2. The method of averaging. In this section, we present the main results on averaging for functional differential equations with delay.

Suppose $f: \mathbb{R} \times U \rightarrow \mathbb{R}^{n}$ is a continuous function, where $U$ is an open subset of $\mathbb{R}^{n}$. Let $\phi:[-r, 0] \rightarrow U$ be a continuous function, where $r>0$ is a constant. Let $\varepsilon>0$ be a real parameter. Along with the functional differential equation with delay

$$
\dot{x}(t)=f\left(\frac{t}{\varepsilon}, x(t-r)\right), \quad \text { for } t>0, \quad x(t)=\phi(t), \quad \text { for } t \in[-r, 0],
$$

we consider the averaged equation

$$
\dot{y}(t)=f^{o}(y(t-r)), \quad \text { for } t>0, \quad y(t)=\phi(t), \quad \text { for } t \in[-r, 0],
$$

where

$$
f^{o}(x):=\lim _{T \rightarrow \infty} \frac{1}{T} \int_{0}^{T} f(t, x) d t .
$$

As a first result, we give a comparison of the solutions of the averaged and the original equations on finite time intervals.

THEOREM 2.1. Assume that

(H.0) The function $f$ is bounded on $\mathbb{R} \times U$.

(H.1) The continuity of $f$ in $x \in U$ is uniform with respect to $t \in \mathbb{R}_{+}$.

(H.2) For all $x \in U$ there exists a limit

$$
f^{o}(x)=\lim _{T \rightarrow \infty} \frac{1}{T} \int_{0}^{T} f(t, x) d t .
$$

(H.3) Equation (2.2) has a unique solution.

Let $y$ be the solution of (2.2) and let $J=[-r, \omega), 0<\omega \leq+\infty$, be its maximal interval of definition. For any $L>0, L \in J$, and any $\delta>0$ there exists an $\varepsilon_{0}=\varepsilon_{0}(L, \delta)>0$ such that, for all $\varepsilon \in\left(0, \varepsilon_{0}\right]$ any solution $x$ of $(2.1)$ is defined at least on $[0, L]$ and satisfies $\|x(t)-y(t)\|<\delta$ for all $t \in[0, L]$.

REMARK 2.2. Assume that the initial time $t_{0} \neq 0$. Let $y\left(\cdot ; t_{0}\right)$ be the solution of (2.2) and let $J=\left[t_{0}-r, t_{0}+\omega\right), 0<\omega \leq+\infty$, be its maximal interval of definition. The conclusions of Theorem 2.1 become for any $L>0, L+t_{0} \in J$, and any $\delta>0$ there exists an $\varepsilon_{0}=\varepsilon_{0}(L, \delta)>0$ such that, for all $\varepsilon \in\left(0, \varepsilon_{0}\right]$ any solution $x\left(\cdot ; t_{0}\right)$ of $(2.1)$ is defined at least on $\left[t_{0}, t_{0}+L\right]$ and satisfies $\left\|x\left(t ; t_{0}\right)-y\left(t ; t_{0}\right)\right\|<\delta$ for all $t \in\left[t_{0}, t_{0}+L\right]$.

One can also precise the long time behaviour of a solution of (2.1) provided that more is known about the solution of (2.2). To give estimate for all time, we assume that the solution of (2.2) tends toward an equilibrium. Before this, we first recall the concept of uniform asymptotic stability of equilibrium points of autonomous functional differential equations with delay.

Consider the autonomous functional differential equation with delay

$$
\dot{y}(t)=f^{o}(y(t-r)),
$$


where $f^{o}: U \rightarrow \mathbb{R}^{n}$ is a continuous function, and $U$ is an open subset of $\mathbb{R}^{n}, r>0$ is a constant. For $t_{0} \in \mathbb{R}$ and $\phi:[-r, 0] \rightarrow \mathbb{R}^{n}$ a continuous function, let $y\left(\cdot ; t_{0}, \phi\right)$ denotes the solution of (2.5) for the initial function $y\left(t ; t_{0}, \phi\right)=\phi(t)$ for $t \in[-r, 0]$.

Since (2.5) is autonomous, the concepts of asymptotic stability and uniform asymptotic stability of equilibrium points of (2.5) coincide. Then, it is sufficient to deal directly with uniform notions.

DEFINITION 2.3. The equilibrium point $y_{e}$ of (2.5) is said to be

(1) Uniformly stable (in the sense of Liapunov) if for any $\mu>0$, there exists $\eta=$ $\eta(\mu)>0$ with the property that for all $t_{0} \in \mathbb{R}$ any solution $y\left(\cdot ; t_{0}, \phi\right)$ of $(2.5)$ for which $\left\|\phi(t)-y_{e}\right\|<\eta$ for $t \in[-r, 0]$, can be continued for all $t>t_{0}$ and satisfies $\left\|y\left(t ; t_{0}, \phi\right)-y_{e}\right\|<\mu$.

(2) Uniformly attractive if there exists $b_{0}>0$ with the respective properties:

(a) For all $t_{0} \in \mathbb{R}$ any solution $y\left(\cdot ; t_{0}, \phi\right)$ of (2.5) for which $\left\|\phi(t)-y_{e}\right\|<b_{0}$ for $t \in[-r, 0]$, can be continued for all $t>t_{0}$.

(b) For every $\delta>0$, there exists $T=T(\delta)>0$ (T depends on $\delta$ but not on $t_{0}$ ) such that $\left\|y\left(t ; t_{0}, \phi\right)-y_{e}\right\|<\delta$ for $t>t_{0}+T(\delta)$, that is, $\lim _{t \rightarrow \infty} y\left(t ; t_{0}, \phi\right)=y_{e}$ uniformly in $t_{0} \in \mathbb{R}$.

(3) Uniformly asymptotically stable if it is uniformly stable and uniformly attractive.

REMARK 2.4. The ball $\mathscr{B}$ of center $y_{e}$ and radius $b_{0}$ where the attraction is uniform will be called the basin of attraction of $y_{e}$.

We now return to the averaged equation (2.2). We assume that $y_{e}$ is an equilibrium point of (2.2), that is, $f^{o}\left(y_{e}\right)=0$. As a next result of this section, we prove the validity of the approximation of a solution $x$ of (2.1) by the solution $y$ of (2.2) for all (future) time, under the additional conditions about the equilibrium point $y_{e}$ and the initial function $\phi$.

THEOREM 2.5. Let the hypotheses (H.O)-(H.3) of Theorem 2.1 be true, and assume that

(H.4) The point $y_{e}$ is uniformly asymptotically stable.

(H.5) The initial function $\phi$ in (2.2) lies in the basin of attraction of $y_{e}$.

Let $y$ be the solution of (2.2). Then for any $\delta>0$ there exists $\varepsilon_{0}=\varepsilon_{0}(\delta)>0$ such that, for all $\varepsilon \in\left(0, \varepsilon_{0}\right]$ any solution $x$ of (2.1) is defined for all $t \geq 0$ and satisfies $\|x(t)-y(t)\|<\delta$ for all $t \geq 0$.

\section{Nonstandard results}

3.1. A short tutorial on Internal Set Theory. Internal Set Theory (IST) is an axiomatic description of nonstandard analysis proposed by Nelson [11]. We complete the ordinary mathematical language (say ZFC) with a new undefined monadic predicate symbol st (read standard). We call internal, the formulas of IST without any occurrence of the predicate st in them; otherwise, we call them external. Thus internal formulas are the formulas of ZFC. The axioms of IST are all axioms of ZFC, restricted to internal formulas (in other words, IST is an extension of ZFC), plus three others which govern the use of the new predicate. Thus all theorems of ZFC remain valid in IST. IST is 
a conservative extension of ZFC, that is, every internal theorem of IST is a theorem of ZFC. There is an algorithm (a well-known reduction algorithm) to reduce any external formula $F\left(x_{1}, \ldots, x_{n}\right)$ of IST without other free variables than $x_{1}, \ldots, x_{n}$, to an internal formula $F^{\prime}\left(x_{1}, \ldots, x_{n}\right)$ with the same free variables, such that $F \equiv F^{\prime}$, that is, $F \Leftrightarrow F^{\prime}$ for all standard values of the free variables. In other words, any result which may be formalized within IST by a formula $F\left(x_{1}, \ldots, x_{n}\right)$ is equivalent to the classical property $F^{\prime}\left(x_{1}, \ldots, x_{n}\right)$, provided the parameters $x_{1}, \ldots, x_{n}$ are restricted to standard values. We give the reduction of the frequently occurring formula $\forall x\left(\forall^{\text {st }} y A \Rightarrow \forall^{\text {st }} z B\right)$ where $A$ and $B$ are internal formulas

$$
\forall x\left(\forall^{\text {st }} y A \Longrightarrow \forall^{\text {st }} z B\right) \equiv \forall z \exists^{\text {fin }} y^{\prime} \forall x\left(\forall y \in y^{\prime} A \Longrightarrow B\right) .
$$

A real number $x$ is called infinitesimal, denoted by $x \simeq 0$, if its absolute value $|x|$ is smaller than any standard strictly positive real number, limited if its absolute value $|x|$ is smaller than some standard real number, unlimited, denoted by $x \simeq \pm \infty$, if it is not limited, and appreciable if it is neither unlimited nor infinitesimal. Two real numbers $x$ and $y$ are infinitely close, denoted by $x \simeq y$, if their difference $x-y$ is infinitesimal.

For $x$ and $y$ in a standard metric space $E$, the notation $x \simeq y$ means that the distance from $x$ to $y$ is infinitesimal. If there exists in that space a standard $x_{0}$ such that $x \simeq x_{0}$, the element $x$ is called nearstandard in $E$ and the standard point $x_{0}$ is called the standard part of $x$ (it is unique) and is also denoted by ${ }^{o} x$. The halo of $x$, denoted by hal $(x)$, is the set, usually external, of all $y$ such that $x \simeq y$. For any subset $X$ of $E$, a point $x \in E$ is called nearstandard in $X$ if there exists a standard point $x_{0} \in X$ such that $x \simeq x_{0}$. We recall that, if $X$ is standard, $X$ is open if and only if it contains the halo of all its standard elements. The shadow of a subset $X$ of $E$, denoted by ${ }^{o} X$, is the unique standard set whose standard elements are precisely those whose halo intersects $X$.

Let $E$ and $F$ be standard metric spaces, and $g$ be an internal function defined on $\mathscr{D}(g) \subset E$ and with values in $F$. The function $g$ is called $S$-continuous at $x_{0} \in \mathscr{D}(g)$ if for all $x \simeq x_{0}, g(x) \simeq g\left(x_{0}\right)$ holds, $S$-continuous in $E \times F$ if it is $S$-continuous at each point $x \in \mathscr{D}(g)$ such that $(x, g(x))$ is nearstandard in $E \times F$. For $g$ standard, the continuity and the $S$-continuity in $\mathscr{D}(g) \times \mathscr{B}(g)$, where $\mathscr{B}(g)$ is a target of $g$, coincide. The shadow in $E \times F$ of the graph of an $S$-continuous function $g$ is the graph of a standard continuous function $g_{0}$, called the shadow of $g$, and denoted by ${ }^{o} g$.

In ZFC in principle all sets are defined using the only nonlogical symbol $\in$. In IST there is also the possibility to define collections with the nonlogical symbol st. Those collections which fall outside the range of ZFC are called external sets. External sets are often easily recognized: mostly some elementary classical property fails to hold. For instance, the set of infinitesimal real numbers hal(0) must be external, for it constitutes a bounded subset of $\mathbb{R}$ without lower upper bound. It happens sometimes in classical mathematics that a property is assumed, or proved, on a certain domain, and that afterwards it is noticed that the character of the property and the nature of the domain are incompatible. So actually the property must be valid on a large domain. In nonstandard analysis, statements which affirm that the validity of a property exceeds the domain where it was established in a direct way are called permanence principles. Many permanence results used in nonstandard analysis are based upon 
the self-evident statement "no external set is internal." This statement is called the Cauchy principle. It has the following frequently used application.

LEMMA 3.1 (Robinson's lemma). If $r$ is an internal real function such that $r(t) \simeq 0$ for all limited $t \geq 0$, then there exists $v \simeq+\infty$ such that $r(t) \simeq 0$ for all $t \in[0, v]$.

Proof. Indeed, $\{l \in \mathbb{R} \mid l \geq 1, l$ limited $\} \subset\{l \in \mathbb{R}|\forall t \in[0, l]| r(t) \mid<1 / l\}$. The first set is external and the second set is internal. By the Cauchy principle the inclusion is strict.

Together with one among its corollaries, we conclude this section with another application of Cauchy's principle which will be used later.

LEMMA 3.2. Let $I$ be an internal set and $h: I \rightarrow \mathbb{R}$ be an internal function such that $h(t) \simeq 0$ for all $t \in I$. Then $\sup _{t \in I}\{h(t)\} \simeq 0$.

Proof. We have $\left\{l \in \mathbb{R}_{+} \mid l \notin \operatorname{hal}(0)\right\} \subset\{l \in \mathbb{R}|\forall t \in I| h(t) \mid<l\}$. The first set is external, otherwise hal $(0)$ would be internal, and the second set is internal. By the Cauchy principle the inclusion is strict.

LEMMA 3.3 (corollary of Lemma 3.2). Let $a<b, b-a$ limited, and let $g, \tilde{g}:[a, b] \rightarrow$ $\mathbb{R}^{n}$ be internal integrable functions such that $g(t) \simeq \tilde{g}(t)$ for all $t \in[a, b]$. Then

$$
\int_{a}^{b} g(t) d t \simeq \int_{a}^{b} \tilde{g}(t) d t
$$

REMARK 3.4. The interested reader is referred to [2, 3, 4, 5, 10, 11, 12, 15, 16] for more informations on nonstandard analysis and its applications.

3.2. The averaging results. First we give nonstandard formulations of Theorem 2.1, Remark 2.2, and Theorem 2.5. Then, by use of the reduction algorithm, we show that the reduction of Theorem 3.5, Remark 3.6, and Theorem 3.7 are Theorem 2.1, Remark 2.2 and Theorem 2.5, respectively.

THEOREM 3.5. Let $U$ be a standard open subset of $\mathbb{R}^{n}$. Let $f: \mathbb{R} \times U \rightarrow \mathbb{R}^{n}$ and $\phi:[-r, 0] \rightarrow U$ be standard continuous functions. Assume that hypotheses (H.O)-(H.3) in Theorem 2.1 hold. Let $y$ be the solution of (2.2) and let $J=[-r, \omega), 0<\omega \leq+\infty$, be its maximal interval of definition. Let $\varepsilon>0$ be infinitesimal. Then for any standard $L>0, L \in J$, any solution $x$ of (2.1) is defined at least on $[0, L]$ and satisfies $x(t) \simeq y(t)$ for all $t \in[0, L]$.

REMARK 3.6. Assume that the initial time $t_{0} \neq 0$. Let $y\left(\cdot ; t_{0}\right)$ be the solution of (2.2) and let $J=\left[t_{0}-r, t_{0}+\omega\right), 0<\omega \leq+\infty$, be its maximal interval of definition. The conclusions of Theorem 3.5 become: let $\varepsilon>0$ be infinitesimal, then for any standard $L>0, L+t_{0} \in J$, any solution $x$ of (2.1) is defined at least on $\left[t_{0}, t_{0}+L\right]$ and satisfies $x(t) \simeq y(t)$ for all $t \in\left[t_{0}, t_{0}+L\right]$.

THEOREM 3.7. Let $U$ be a standard open subset of $\mathbb{R}^{n}$. Let $f: \mathbb{R} \times U \rightarrow \mathbb{R}^{n}$ and $\phi:[-r, 0] \rightarrow U$ be standard continuous functions. Let $y_{e}$ be a standard equilibrium point of (2.2). Assume that hypotheses (H.O)-(H.5) in Theorem 2.5 hold. Let $y$ be the 
solution of (2.2). Let $\varepsilon>0$ be infinitesimal. Then any solution $x$ of (2.1) is defined for all $t \geq 0$ and satisfies $x(t) \simeq y(t)$ for all $t \geq 0$.

The proofs of Theorems 3.5 and 3.7 are postponed to Section 4. Theorem 3.5, Remark 3.6, and Theorem 3.7 are external statements. We show that the reduction of Theorem 3.5 (resp., Remark 3.6 and Theorem 3.7) is Theorem 2.1 (resp., Remark 2.2 and Theorem 2.5).

REDUCTION OF THEOREM 3.5. Let $B$ be the formula "if $\delta>0$ then any solution $x$ of (2.1) is defined at least on $[0, L]$ and satisfies $\|x(t)-y(t)\|<\delta$ for all $t \in[0, L]$ ". To say that "any solution $x$ of (2.1) is defined at least on $[0, L]$ and satisfies $x(t) \simeq y(t)$ for all $t \in[0, L]$ " is the same as saying $\forall^{\text {st }} \delta B$. Then Theorem 3.5 asserts that

$$
\forall \varepsilon\left(\forall^{\mathrm{st}} \eta \varepsilon<\eta \Longrightarrow \forall^{\mathrm{st}} \delta B\right) .
$$

In this formula $L$ is standard and $\varepsilon, \eta$, and $\delta$ range over the strictly positive real numbers. By (3.1), formula (3.3) is equivalent to

$$
\forall \delta \exists^{\text {fin }} \eta^{\prime} \forall \varepsilon\left(\forall \eta \in \eta^{\prime} \varepsilon<\eta \Longrightarrow B\right) .
$$

For $\eta^{\prime}$ a finite set, for all $\eta \in \eta^{\prime} \varepsilon<\eta$ is the same as $\varepsilon<\varepsilon_{0}$ for $\varepsilon_{0}=\min \eta^{\prime}$, and so formula (3.4) is equivalent to

$$
\forall \delta \exists \varepsilon_{0} \forall \varepsilon\left(\varepsilon<\varepsilon_{0} \Longrightarrow B\right) .
$$

This shows that for any standard $L>0, L \in J$, the statement of Theorem 2.1 holds, thus by transfer, it holds for any $L>0, L \in J$.

The reduction of Remark 3.6 (resp., Theorem 3.7) to Remark 2.2 (resp., Theorem 2.5) follows almost verbatim the reduction of Theorem 3.5 to Theorem 2.1 and is left to the reader.

3.3. Uniform asymptotic stability. As the condition (H.4) will be used in its external form, we give the external characterizations of the notion of uniform stability and uniform attractiveness of the equilibrium point $y_{e}$ of (2.5), given in Definition 2.3.

By transfer, we may assume that $f^{o}, r$, and $y_{e}$ are standard.

LEMMA 3.8. The equilibrium point $y_{e}$ of (2.5) is

(1) Uniformly stable if and only if for all $t_{0} \in \mathbb{R}$ any solution $y\left(\cdot ; t_{0}, \phi\right)$ of (2.5) for which $\phi(t) \simeq y_{e}$ for $t \in[-r, 0]$, can be continued for all $t>t_{0}$ and satisfies $y\left(t ; t_{0}, \phi\right) \simeq y_{e}$.

(2) Uniformly attractive if and only if it admits a standard basin of attraction, that is, there exists a standard $b_{0}>0$ with the property that for all $t_{0} \in \mathbb{R}$ any solution $y\left(\cdot ; t_{0}, \phi\right)$ of (2.5) for which $\left\|\phi(t)-y_{e}\right\|<b_{0}$ for $t \in[-r, 0]$, $\phi$ standard, can be continued for all $t>t_{0}$ and satisfies $y\left(t ; t_{0}, \phi\right) \simeq y_{e}$ for all $t$ such that $t-t_{0} \simeq+\infty$.

Proof. (1) Let $A$ be the formula " $\left\|\phi(t)-y_{e}\right\| \mid<\eta$ for $t \in[-r, 0]$ " and let $C$ be the formula "Any solution $y\left(\cdot ; t_{0}, \phi\right)$ of (2.5) can be continued for all $t>t_{0}$ and satisfies the inequality $\left\|y\left(t ; t_{0}, \phi\right)-y_{e}\right\|<\mu$." The characterization of uniform stability in the lemma is

$$
\forall t_{0} \forall \phi\left(\forall^{\mathrm{st}} \eta A \Longrightarrow \forall^{\mathrm{st}} \mu B\right)
$$


In this formula $y(\cdot ; \cdot, \cdot), r$, and $y_{e}$ are standard parameters and $\eta, \mu$ range over the strictly positive real numbers. By (3.1), formula (3.6) is equivalent to

$$
\forall \mu \exists^{\text {fin }} \eta^{\prime} \forall t_{0} \forall \phi\left(\forall \eta \in \eta^{\prime} A \Longrightarrow B\right) .
$$

For $\eta^{\prime}$ a finite set, for all $\eta \in \eta^{\prime} A$ is the same as $A$ for $\eta=\min \eta^{\prime}$, and so formula (3.7) is equivalent to

$$
\forall \mu \exists \eta \forall t_{0} \forall \phi(A \Longrightarrow B) .
$$

This is the usual definition of uniform stability.

(2) By transfer, the uniform attractiveness of $y_{e}$ is equivalent to the existence of a standard basin of attraction, that is, $b_{0}$ in Remark 2.4 is standard. The characterization of standard basin of attraction in the lemma is that for all standard continuous function $\phi:[-r, 0] \rightarrow U$ such that $\left\|\phi(t)-y_{e}\right\|<b_{0}$ for $t \in[-r, 0]$, we have the property that for all $t_{0} \in \mathbb{R}$ any solution $y\left(\cdot ; t_{0}, \phi\right)$ of (2.5) can be continued for all $t>t_{0}$ and satisfies

$$
\forall t_{0} \forall t\left(\forall^{\mathrm{st}} T t-t_{0}>T \Longrightarrow \forall^{\mathrm{st}} \delta\left\|y\left(t ; t_{0}, \phi\right)-y_{e}\right\|<\delta\right) .
$$

In this formula $y(\cdot ; \cdot, \phi)$ and $y_{e}$ are standard parameters and $T, \delta$ range over the strictly positive real numbers. By (3.1), formula (3.9) is equivalent to

$$
\forall \delta \exists^{\mathrm{fin}} T^{\prime} \forall t_{0} \forall t\left(\forall T \in T^{\prime} t-t_{0}>T \Longrightarrow\left\|y\left(t ; t_{0}, \phi\right)-y_{e}\right\|<\delta\right) .
$$

For $T^{\prime}$ a finite set for all $T \in T^{\prime} t-t_{0}>T$ is the same as $t-t_{0}>T$ for $T=\max T^{\prime}$, and so formula (3.10) is equivalent to

$$
\forall \delta \exists T \forall t_{0} \forall t\left(t-t_{0}>T \Longrightarrow\left\|y\left(t ; t_{0}, \phi\right)-y_{e}\right\|<\delta\right) .
$$

We have shown that for all standard continuous function $\phi$ in the basin of attraction of $y_{e}$ (and consequently, by transfer, for all continuous function $\phi$ in the basin of attraction of $\left.y_{e}\right)$, for all $t_{0} \in \mathbb{R}$ any solution $y\left(\cdot ; t_{0}, \phi\right)$ of (2.5) can be continued for all $t>t_{0}$ and satisfies $\lim _{t \rightarrow \infty} y\left(t ; t_{0}, \phi\right)=y_{e}$, the limit being uniform in $t_{0}$.

Assume that (2.5) has the uniqueness of the solutions with prescribed initial functions. Let $\phi:[-r, 0] \rightarrow U$ be continuous. For $t_{0} \in \mathbb{R}$, let $y\left(\cdot ; t_{0}, \phi\right)$ be the solution of (2.5) for initial function $y\left(t ; t_{0}, \phi\right)=\phi(t)$ for $t \in[-r, 0]$. This solution is defined on the interval $I\left(t_{0}, \phi\right)=\left[t_{0}-r, t_{0}+\beta\right)$. It is well known that the function $y$ is continuous with respect to the initial function $\phi$. The external formulation of this result is as follows.

LEMMA 3.9. Let $\phi$ and $\phi_{0}:[-r, 0] \rightarrow U$ be continuous, with $\phi_{0}$ standard. If $\phi(t) \simeq$ $\phi_{0}(t)$ on $[-r, 0]$, then for all $t \in I\left(t_{0}, \phi_{0}\right), t>t_{0}$, such that $t-t_{0}$ is standard, we have $t \in I\left(t_{0}, \phi\right)$ and $y\left(t ; t_{0}, \phi\right) \simeq y\left(t ; t_{0}, \phi_{0}\right)$.

Proof. The reduction of Lemma 3.9 is the usual continuity of the solutions with respect to the initial functions.

LEMMA 3.10. Assume that (2.5) has the uniqueness of the solutions with the prescribed initial functions. The equilibrium point $y_{e}$ of (2.5) is uniformly asymptotically 
stable if and only if there exists a standard $a>0$ with the property that for all $t_{0} \in \mathbb{R}$ any solution $y\left(\cdot ; t_{0}, \phi\right)$ of (2.5) for which $\left\|\phi(t)-y_{e}\right\|<a$ for $t \in[-r, 0]$, can be continued for all $t>t_{0}$ and satisfies $y\left(t ; t_{0}, \phi\right) \simeq y_{e}$ for all $t$ such that $t-t_{0} \simeq+\infty$.

Proof. Assume that $y_{e}$ is uniformly asymptotically stable. Then it is uniformly attractive, and so it admits a ball $\mathscr{B}$ of center $y_{e}$ and radius $b_{0}>0, b_{0}$ standard, as a standard basin of attraction. Let $a>0$ be standard such that the closure of the ball $\mathscr{R}^{\prime}$ of center $y_{e}$ and radius $a$ is included in $\mathscr{B}$. Let $\phi$ and $\phi_{0}:[-r, 0] \rightarrow U$ be continuous, $\phi_{0}$ standard, with $\phi(t) \in \mathscr{B}^{\prime}$ and $\phi_{0}(t) \in \mathscr{B}$ for all $t \in[-r, 0]$. Assume that $\phi(t) \simeq$ $\phi_{0}(t)$ for all $t \in[-r, 0]$. For $t_{0} \in \mathbb{R}$, let $y=y\left(\cdot ; t_{0}, \phi\right)$ and $y_{0}=y_{0}\left(\cdot ; t_{0}, \phi_{0}\right)$ be the solutions of (2.5) with the initial functions $\phi$ and $\phi_{0}$, respectively. By the uniform attractiveness of $y_{e}$, the solution $y_{0}$ is defined for all $t>t_{0}$ and satisfies $y_{0}(t) \simeq y_{e}$ for all $t-t_{0} \simeq+\infty$. By Lemma 3.9, $y(t) \simeq y_{0}(t)$ on $\left[t_{0}, t_{0}+L\right]$ for all limited $L>0$. By Robinson's lemma, there exists $v \simeq+\infty$ such that $y(t) \simeq y_{0}(t)$ on $\left[t_{0}, t_{0}+v\right]$. Thus $y(t) \simeq y_{e}$ for all $t \leq t_{0}+v, t-t_{0} \simeq+\infty$, and in particular we have $y(t) \simeq y_{e}$ for all $t \in\left[t_{0}+v-r, t_{0}+v\right]$. By the uniform stability of $y_{e}$ we have $y(t) \simeq y_{e}$ for all $t>t_{0}+v$. Hence $y(t) \simeq y_{e}$ for all $t$ such that $t-t_{0} \simeq+\infty$. Conversely, assume $y_{e}$ satisfies the property in the lemma. By Lemma 3.8(2), the ball $\mathscr{B}^{\prime}$ is a standard basin of attraction of $y_{e}$. Hence $y_{e}$ is uniformly attractive. Let $\phi:[-r, 0] \rightarrow U$ be continuous with $\phi(t) \simeq y_{e}$ for all $t \in[-r, 0]$. For $t_{0} \in \mathbb{R}$, let $y\left(\cdot ; t_{0}, \phi\right)$ be the solution of (2.5). By hypothesis we have $y\left(t ; t_{0}, \phi\right) \simeq y_{e}$ for all $t$ such that $t-t_{0} \simeq+\infty$, and by Lemma 3.9, $y\left(t ; t_{0}, \phi\right) \simeq y\left(t ; t_{0}, y_{e}\right)=y_{e}$ for all $t$ such that $t-t_{0}$ is limited. By Lemma 3.8(1), $y_{e}$ is uniformly stable. Thus $y_{e}$ is uniformly asymptotically stable.

\section{Proofs of Theorems 3.5 and 3.7}

4.1. Preliminary lemmas. Hereafter we give some results we need for the proof of Theorem 3.5. We assume throughout this section that $f$ and $\phi$ are standard. We suppose also that $f$ satisfies conditions (H.0), (H.1), and (H.2) of Theorem 3.5. The conditions (H.1) and (H.2) will be used in their following external forms

(H.1') $\forall^{\mathrm{st}} x_{0} \in U \forall t>0 \forall x \in U\left(x \simeq x_{0} \Rightarrow f(t, x) \simeq f\left(t, x_{0}\right)\right)$.

(H.2') There is a standard function $f^{o}: U \rightarrow \mathbb{R}^{n}$ such that

$$
\forall^{\mathrm{st}} x_{0} \in U \quad \forall T \simeq+\infty \quad\left(f^{o}\left(x_{0}\right) \simeq \frac{1}{T} \int_{0}^{T} f\left(t, x_{0}\right) d t\right) .
$$

LEMмA 4.1. The function $f^{o}$ is continuous and we have

$$
f^{o}(x) \simeq \frac{1}{T} \int_{0}^{T} f(t, x) d t
$$

for all $x$ nearstandard in $U$ and all $T \simeq+\infty$.

Proof. See [14, Lemma 4, page 106].

LEMmA 4.2. For all limited $t>0$ and all $x$ nearstandard in $U$, there is $\alpha>0, \alpha \simeq 0$, such that

$$
\frac{\varepsilon}{\alpha} \int_{t / \varepsilon}^{t / \varepsilon+\alpha / \varepsilon} f(\tau, x) d \tau \simeq f^{o}(x) .
$$


Proof. See [14, Lemma 5, page 107], with $T=1, S=\alpha / \varepsilon$ and $s=t / \varepsilon$.

LEMMA 4.3. Let $L_{1}>0$ be standard and let $\tilde{x}$ be a function defined on $\left[-r, L_{1}\right]$. We assume that $\tilde{x}$ is continuous on $[-r, 0], \tilde{x}(t)$ is nearstandard in $U$ for all $t \in\left[0, L_{1}\right]$, and satisfies $\tilde{x}(t) \simeq \tilde{x}\left(t_{n}\right)$ for all $t \in\left[t_{n}, t_{n+1}\right]$ with $0=t_{0}<\cdots<t_{n}<t_{n+1}<\cdots<$ $t_{\omega} \leq L_{1}<t_{\omega+1}$ and $t_{n+1}-t_{n}=\alpha_{n} \simeq 0$, where $\alpha_{n}$ is determined by Lemma 4.2. Then

$$
\int_{0}^{t} f\left(\frac{\tau}{\varepsilon}, \tilde{x}(\tau-r)\right) d \tau \simeq \int_{0}^{t} f^{o}(\tilde{x}(\tau-r)) d \tau \quad \forall t \in\left[0, L_{1}\right]
$$

Proof. Let $t \in\left[0, L_{1}\right]$ and let $N$ be a positive integer such that $t_{N} \leq t<t_{N+1}$. We have

$$
\begin{aligned}
\int_{0}^{t} f( & \left.\frac{\tau}{\varepsilon}, \tilde{x}(\tau-r)\right) d \tau-\int_{0}^{t} f^{o}(\tilde{x}(\tau-r)) d \tau \\
= & \int_{0}^{t}\left(f\left(\frac{\tau}{\varepsilon}, \tilde{x}(\tau-r)\right)-f^{o}(\tilde{x}(\tau-r))\right) d \tau \\
= & \sum_{n=0}^{N-1} \int_{t_{n}}^{t_{n+1}}\left(f\left(\frac{\tau}{\varepsilon}, \tilde{x}(\tau-r)\right)-f^{o}(\tilde{x}(\tau-r))\right) d \tau \\
& +\int_{t_{N}}^{t}\left(f\left(\frac{\tau}{\varepsilon}, \tilde{x}(\tau-r)\right)-f^{o}(\tilde{x}(\tau-r))\right) d \tau \\
\simeq & \sum_{n=0}^{N-1} \int_{t_{n}}^{t_{n+1}}\left(f\left(\frac{\tau}{\varepsilon}, \tilde{x}(\tau-r)\right)-f^{o}(\tilde{x}(\tau-r))\right) d \tau,
\end{aligned}
$$

since

$$
\begin{gathered}
\left\|\int_{t_{N}}^{t}\left(f\left(\frac{\tau}{\varepsilon}, \tilde{x}(\tau-r)\right)-f^{o}(\tilde{x}(\tau-r))\right) d \tau\right\| \\
\quad \leq \int_{t_{N}}^{t}\left(\left\|f\left(\frac{\tau}{\varepsilon}, \tilde{x}(\tau-r)\right)\right\|+\left\|f^{o}(\tilde{x}(\tau-r))\right\|\right) d \tau \\
\quad \leq 2 M\left(t-t_{N}\right) \leq 2 M\left(t_{N+1}-t_{N}\right) \leq 2 M \alpha \simeq 0,
\end{gathered}
$$

where $\alpha=\max \left\{\alpha_{n}\right\} \simeq 0$ (see Lemma 3.2) and $M$ is a bound for $f$ and then for $f^{o}$ too, $M$ is standard.

On the other hand, it is easy to verify that $\tilde{x}(\tau-r) \simeq \tilde{x}\left(t_{n}-r\right)=$ cte $:=\tilde{x}_{n}$ for $\tau \in\left[t_{n}, t_{n+1}\right]$. By the continuity of $f$, condition (H.1'), and Lemma 4.1 (the continuity of $f^{o}$ ) it follows, respectively, that

$$
f\left(\frac{\tau}{\varepsilon}, \tilde{x}(\tau-r)\right) \simeq f\left(\frac{\tau}{\varepsilon}, \tilde{x}_{n}\right), \quad f^{o}(\tilde{x}(\tau-r)) \simeq f^{o}\left(\tilde{x}_{n}\right),
$$

or

$$
f\left(\frac{\tau}{\varepsilon}, \tilde{x}(\tau-r)\right)=f\left(\frac{\tau}{\varepsilon}, \tilde{x}_{n}\right)+\gamma_{n}(\tau), \quad f^{o}(\tilde{x}(\tau-r))=f^{o}\left(\tilde{x}_{n}\right)+\delta_{n}(\tau),
$$


with

$$
\gamma_{n}(\tau) \simeq 0 \simeq \delta_{n}(\tau)
$$

Hence, from (4.5), we obtain

$$
\begin{aligned}
\int_{0}^{t} f( & \left.\frac{\tau}{\varepsilon}, \tilde{x}(\tau-r)\right) d \tau-\int_{0}^{t} f^{o}(\tilde{x}(\tau-r)) d \tau \\
\simeq & \sum_{n=0}^{N-1} \int_{t_{n}}^{t_{n+1}}\left(f\left(\frac{\tau}{\varepsilon}, \tilde{x}_{n}\right)-f^{o}\left(\tilde{x}_{n}\right)+\gamma_{n}(\tau)+\delta_{n}(\tau)\right) d \tau \\
= & \sum_{n=0}^{N-1} \int_{t_{n}}^{t_{n+1}}\left(f\left(\frac{\tau}{\varepsilon}, \tilde{x}_{n}\right)-f^{o}\left(\tilde{x}_{n}\right)\right) d \tau+\sum_{n=0}^{N-1} \int_{t_{n}}^{t_{n+1}}\left(\gamma_{n}(\tau)+\delta_{n}(\tau)\right) d \tau \\
\simeq & \sum_{n=0}^{N-1} \int_{t_{n}}^{t_{n+1}}\left(f\left(\frac{\tau}{\varepsilon}, \tilde{x}_{n}\right)-f^{o}\left(\tilde{x}_{n}\right)\right) d \tau+\sum_{n=0}^{N-1}\left(\gamma_{n}+\delta_{n}\right) \int_{t_{n}}^{t_{n+1}} d \tau \\
\simeq & \sum_{n=0}^{N-1} \int_{t_{n}}^{t_{n+1}}\left(f\left(\frac{\tau}{\varepsilon}, \tilde{x}_{n}\right)-f^{o}\left(\tilde{x}_{n}\right)\right) d \tau+(\gamma+\delta) \sum_{n=0}^{N-1}\left(t_{n+1}-t_{n}\right) \\
& =\sum_{n=0}^{N-1} \int_{t_{n}}^{t_{n+1}}\left(f\left(\frac{\tau}{\varepsilon}, \tilde{x}_{n}\right)-f^{o}\left(\tilde{x}_{n}\right)\right) d \tau+(\gamma+\delta) \cdot t_{N}
\end{aligned}
$$

with $\gamma_{n}(\tau) \simeq \delta_{n}(\tau) \simeq 0$ for $\tau \in\left[t_{n}, t_{n+1}\right]$ and where $\gamma_{n}+\delta_{n}=\sup _{\tau \in\left[t_{n}, t_{n+1}\right]}\{\gamma(\tau)\}+$ $\sup _{\tau \in\left[t_{n}, t_{n+1}\right]}\{\delta(\tau)\} \simeq 0$ and $\gamma+\delta=\max \left\{\gamma_{n}\right\}+\max \left\{\delta_{n}\right\} \simeq 0$ (see Lemma 3.2).

Since $L_{1}$ is standard and $t_{N} \in\left[0, L_{1}\right], t_{N}$ is limited and then $(\gamma+\delta) \cdot t_{N} \simeq 0$. Therefore,

$$
\int_{0}^{t} f\left(\frac{\tau}{\varepsilon}, \tilde{x}(\tau-r)\right) d \tau-\int_{0}^{t} f^{o}(\tilde{x}(\tau-r)) d \tau \simeq \sum_{n=0}^{N-1} \int_{t_{n}}^{t_{n+1}}\left(f\left(\frac{\tau}{\varepsilon}, \tilde{x}_{n}\right)-f^{o}\left(\tilde{x}_{n}\right)\right) d \tau .
$$

By Lemma 4.2, we have

$$
\begin{aligned}
\int_{t_{n}}^{t_{n+1}}\left(f\left(\frac{\tau}{\varepsilon}, \tilde{x}_{n}\right)-f^{o}\left(\tilde{x}_{n}\right)\right) d \tau & =\int_{t_{n}}^{t_{n}+\alpha_{n}}\left(f\left(\frac{\tau}{\varepsilon}, \tilde{x}_{n}\right)-f^{o}\left(\tilde{x}_{n}\right)\right) d \tau \\
& =\int_{t_{n}}^{t_{n}+\alpha_{n}} f\left(\frac{\tau}{\varepsilon}, \tilde{x}_{n}\right) d \tau-\int_{t_{n}}^{t_{n}+\alpha_{n}} f^{o}\left(\tilde{x}_{n}\right) d \tau \\
& =\int_{t_{n}}^{t_{n}+\alpha_{n}} f\left(\frac{\tau}{\varepsilon}, \tilde{x}_{n}\right) d \tau-\alpha_{n} \cdot f^{o}\left(\tilde{x}_{n}\right) \\
& =\varepsilon \int_{t_{n} / \varepsilon}^{t_{n} / \varepsilon+\alpha_{n} / \varepsilon} f\left(s, \tilde{x}_{n}\right) d s-\alpha_{n} \cdot f^{o}\left(\tilde{x}_{n}\right), \quad \text { where } s=\frac{\tau}{\varepsilon} \\
& =\alpha_{n}\left(\frac{\varepsilon}{\alpha_{n}} \int_{t_{n} / \varepsilon}^{t_{n} / \varepsilon+\alpha_{n} / \varepsilon} f\left(s, \tilde{x}_{n}\right) d s-f^{o}\left(\tilde{x}_{n}\right)\right) \\
& =\alpha_{n} \cdot \beta_{n}, \quad \text { with } \beta_{n} \simeq 0 .
\end{aligned}
$$


Therefore

$$
\begin{aligned}
\int_{0}^{t} f\left(\frac{\tau}{\varepsilon}, \tilde{x}(\tau-r)\right) d \tau-\int_{0}^{t} f^{o}(\tilde{x}(\tau-r)) d \tau & \simeq \sum_{n=0}^{N-1} \alpha_{n} \cdot \beta_{n} \\
& \simeq \beta \sum_{n=0}^{N-1} \alpha_{n}, \quad \text { where } \beta=\max \left\{\beta_{n}\right\} \\
& =\beta \sum_{n=0}^{N-1}\left(t_{n+1}-t_{n}\right)=\beta \cdot t_{N} .
\end{aligned}
$$

By Lemma 3.2, $\beta \simeq 0$ and then $\beta \cdot t_{N} \simeq 0$. This implies the lemma.

LEMMA 4.4. Let $L_{1}>0$ be standard and let $x$ be a solution of (2.1). Assume that $\left[0, L_{1}\right] \subset I$ and $x(t)$ is nearstandard for all $t \in\left[0, L_{1}\right]$. Then $x$ is $S$-continuous on $\left[0, L_{1}\right]$ and its shadow on $\left[0, L_{1}\right]$ coincides with the solution $y$ of (2.2) on this interval, and satisfies $x(t) \simeq y(t)$ for all $t \in\left[0, L_{1}\right]$.

Proof. The solution $x$ is given as

$$
x(t)=\phi(0)+\int_{0}^{t} f\left(\frac{\tau}{\varepsilon}, x(\tau-r)\right) d \tau, \text { for } t \in\left[0, L_{1}\right] .
$$

As $f$ is bounded on $\mathbb{R} \times U$, it is clear that $x$ is $S$-continuous on $\left[0, L_{1}\right]$.

Let $\tilde{x}$ be a function which satisfies the hypotheses of Lemma 4.3 and such that

$$
x(t) \simeq \tilde{x}(t), \quad \forall t \in\left[-r, L_{1}\right] .
$$

Consider now the following equality which is always true

$$
\begin{aligned}
\int_{0}^{t} f( & \left.\frac{\tau}{\varepsilon}, x(\tau-r)\right) d \tau-\int_{0}^{t} f^{o}(x(\tau-r)) d \tau \\
= & \int_{0}^{t}\left(f\left(\frac{\tau}{\varepsilon}, x(\tau-r)\right)-f\left(\frac{\tau}{\varepsilon}, \tilde{x}(\tau-r)\right)\right) d \tau \\
& +\int_{0}^{t}\left(f\left(\frac{\tau}{\varepsilon}, \tilde{x}(\tau-r)\right)-f^{o}(\tilde{x}(\tau-r))\right) d \tau \\
& +\int_{0}^{t}\left(f^{o}(\tilde{x}(\tau-r))-f^{o}(x(\tau-r))\right) d \tau .
\end{aligned}
$$

As $x(t)$ is nearstandard in $U$ for any $t \in\left[-r, L_{1}\right]$, by the continuity of $f$, condition (H.1') and (4.15), we have

$$
f\left(\frac{t}{\varepsilon}, x(t-r)\right) \simeq f\left(\frac{t}{\varepsilon}, \tilde{x}(t-r)\right)
$$

and by Lemma 3.3,

$$
\int_{0}^{t} f\left(\frac{\tau}{\varepsilon}, x(\tau-r)\right) d \tau \simeq \int_{0}^{t} f\left(\frac{\tau}{\varepsilon}, \tilde{x}(\tau-r)\right) d \tau .
$$


On the other hand, by Lemma 4.1 (the continuity of $f^{o}$ ) and by (4.15), we have

$$
f^{o}(\tilde{x}(t-r)) \simeq f^{o}(x(t-r))
$$

and by Lemma 3.3, we obtain

$$
\int_{0}^{t} f^{o}(\tilde{x}(\tau-r)) d \tau \simeq \int_{0}^{t} f^{o}(x(\tau-r)) d \tau .
$$

By Lemma 4.3 , for any $t \in\left[0, L_{1}\right]$, we have

$$
\int_{0}^{t} f\left(\frac{\tau}{\varepsilon}, \tilde{x}(\tau-r)\right) d \tau \simeq \int_{0}^{t} f^{o}(\tilde{x}(\tau-r)) d \tau .
$$

Hence, for $t \in\left[0, L_{1}\right]$, (4.16) implies

$$
\int_{0}^{t} f\left(\frac{\tau}{\varepsilon}, x(\tau-r)\right) d \tau \simeq \int_{0}^{t} f^{o}(x(\tau-r)) d \tau .
$$

Using (4.14) and (4.22), we obtain

$$
\begin{aligned}
x(t) & =\phi(0)+\int_{0}^{t} f\left(\frac{\tau}{\varepsilon}, x(\tau-r)\right) d \tau \\
& \simeq \phi(0)+\int_{0}^{t} f^{o}(x(\tau-r)) d \tau .
\end{aligned}
$$

Let ${ }^{o} x$ be the shadow of $x$ on $\left[0, L_{1}\right]$. It is easy to see that the function $z$, where

$$
z(t)= \begin{cases}{ }^{o} x(t), & \text { for } t \in\left[0, L_{1}\right], \\ \phi(t), & \text { for } t \in[-r, 0],\end{cases}
$$

is a solution of (2.2). The hypothesis (H.3) insures that $z=y$ on $\left[-r, L_{1}\right]$. Hence, we have $x(t) \simeq y(t)$ for $t \in\left[0, L_{1}\right]$.

Proof of TheOrem 3.5. Let $L>0$ be standard in $J$. Since $\Gamma=y([0, L])$ is a standard compact subset of $U$, there exists $\rho>0, \rho$ standard, and $K$, a standard compact neighborhood of $\Gamma$ included in $U$, such that $\operatorname{dist}(\Gamma, K)=\inf \{\|y-z\| / y \in \Gamma, z \in$ $\left.\mathbb{R}^{n}-K\right\}>\rho$. Let $x: I \rightarrow U$ be a solution of (2.1). Define the set $A=\left\{L_{1} \in I \cap[0, L] \mid\right.$ $\left.x\left(\left[0, L_{1}\right]\right) \subset K\right\}$. The set $A$ is nonempty $(0 \in A)$ and bounded above by $L$. Let $L_{0}$ be a lower upper bound of $A$. There is $L_{1} \in A$ such that $L_{0}-\varepsilon<L_{1} \leq L_{0}$. Thus $x\left(\left[0, L_{1}\right]\right) \subset K$. Hence on $\left[0, L_{1}\right]$ the function $x$ is nearstandard in $U$. By Lemma 4.4 , we have $x(t) \simeq y(t)$ for $t \in\left[0, L_{1}\right]$. Consider the interval $\left[0, L_{1}+\varepsilon\right]$. Let $t \in\left[0, L_{1}+\varepsilon\right]$. As $t-r$ is in $\left[-r, L_{1}+\varepsilon-r\right] \subset\left[-r, L_{1}\right], x(t-r)$ is defined and so is

$$
x(t)=\phi(0)+\int_{0}^{t} f\left(\frac{\tau}{\varepsilon}, x(\tau-r)\right) d \tau .
$$

On the other hand, we have, for $t \in\left[L_{1}, L_{1}+\varepsilon\right], x(t) \simeq x\left(L_{1}\right) \simeq y\left(L_{1}\right)$ with $y\left(L_{1}\right)$ nearstandard in $U$. Hence, on $\left[L_{1}, L_{1}+\varepsilon\right], x$ is nearstandard in $U$. Thus, on $\left[0, L_{1}+\varepsilon\right]$, $x$ is defined and nearstandard in $U$. By Lemma 4.4, we have $x(t) \simeq y(t)$ for $t \in\left[0, L_{1}+\right.$ $\varepsilon]$. Hence $\left[0, L_{1}+\varepsilon\right] \subset I$ and $x\left(\left[0, L_{1}+\varepsilon\right]\right) \subset K$. Suppose $L_{1}+\varepsilon \leq L$, then $L_{1}+\varepsilon \in A$ which is a contradiction. Thus $L_{1}+\varepsilon>L$, that is, we have $x(t) \simeq y(t)$ for all $t \in$ $[0, L] \subset\left[0, L_{1}+\varepsilon\right]$. 
Proof of TheOrem 3.7. By condition (H.5) and the uniform attractiveness of $y_{e}$ (see Lemma 3.8(2)), the solution $y$ is defined for all $t>0$ and satisfies $y(t) \simeq y_{e}$ for all $t \simeq+\infty$. Let $x: I \rightarrow U$ be a solution of (2.1). By Theorem 3.5, for all limited $L>0$, $x$ is defined on $[0, L]$ and the approximation $x(t) \simeq y(t)$ holds for all $t \in[0, L]$. By Robinson's lemma, there exists $t_{1} \simeq+\infty$ such that $x(t) \simeq y(t)$ on $\left[0, t_{1}\right]$. And then we have

$$
x(t) \simeq y(t) \simeq y_{e}, \quad \forall t \leq t_{1}, t \simeq+\infty .
$$

It remains to prove that $x$ is defined for all $t \geq t_{1}$ and satisfies $x(t) \simeq y(t)$ for all $t>t_{1}$. Assume that this is false. Then there exists $s>t_{1}$ such that $x(s) \neq y(s)$, that is,

$$
\|x(s)-y(s)\|=\frac{2}{3} \kappa
$$

is appreciable. Since $y(t) \simeq y_{e}$ for all $t \simeq+\infty$, we have

$$
\left\|x(s)-y_{e}\right\| \leq\|x(s)-y(s)\|+\left\|y(s)-y_{e}\right\| \leq \frac{2}{3} \kappa+\frac{\kappa}{3}=\kappa .
$$

Let $\mathscr{B}$, the ball of center $y_{e}$ and radius $b_{0}>0, b_{0}$ standard, be the basin of attraction of $y_{e}$. We can choose $s$ in (4.28) so that the ball $\mathscr{B}^{\prime}$ of center $y_{e}$ and radius $\kappa$ is included in $\mathscr{B}$, with $b_{0}-\kappa$ appreciable. Let $t_{2}$ be the first instant in time such that equality (4.27) holds. Clearly $t_{2}>t_{1}$.

CASE $1\left(t_{1,2}=t_{2}-t_{1} \simeq+\infty\right)$. Redefine in (2.2) the initial time $r=t_{0}$. Let $z_{1}\left(\cdot ; r, x, t_{2}\right)$ denote the solution of (2.2) such that $z_{1}\left(t ; r, x, t_{2}\right)=x\left(t_{2}-t\right)$ for $t \in[0, r]$. By Theorem 3.5 and Remark 3.6, for all limited $L>0, z_{1}\left(\cdot ; r, x, t_{2}\right)$ is defined on $[r, r+L]$ and satisfies $z_{1}\left(t ; r, x, t_{2}\right) \simeq x\left(t_{2}-t\right)$ for $t \in[r, r+L]$. By Robinson's lemma, there exists $\bar{t}_{1,2} \simeq+\infty$, which one can choose such that $r+\bar{t}_{1,2} \leq t_{1,2}$, with the property that $z_{1}\left(t ; r, x, t_{2}\right) \simeq x\left(t_{2}-t\right)$ on $\left[r, r+\bar{t}_{1,2}\right]$. In particular, $z_{1}\left(t ; r, x, t_{2}\right) \simeq x\left(t_{2}-t\right)$ on $\left[\bar{t}_{1,2}, r+\bar{t}_{1,2}\right] \subset\left[0, t_{1,2}\right]$. Since $x(t)$ belongs to $\mathscr{B}^{\prime}$ for all $t \in\left[t_{1}, t_{2}\right], x\left(t_{2}-t\right)$ lies in $\mathscr{B}^{\prime}$ for all $t \in\left[0, t_{1,2}\right]$. This implies that $z_{1}\left(t ; r, x, t_{2}\right)$ is in $\mathscr{B}$ for all $t \in\left[\bar{t}_{1,2}, r+\bar{t}_{1,2}\right]$. By the uniform attractiveness of $y_{e}$ (see Lemma 3.10), through the transformation $t \mapsto-t$, the solution of (2.2) with the initial function $z_{1}\left(-t ; r, x, t_{2}\right)$ for $t \in\left[-r-\bar{t}_{1,2},-\bar{t}_{1,2}\right]$ which coincides with $z_{1}\left(\cdot ; r, x, t_{2}\right)$ (by uniqueness; hypothesis (H.3)) is defined for all $t>-\bar{t}_{1,2}$ and satisfies $z_{1}\left(-t ; r, x, t_{2}\right) \simeq y_{e}$ for $t+\bar{t}_{1,2} \simeq+\infty$. Take $t=0$, then $x\left(t_{2}\right) \simeq z_{1}\left(0 ; r, x, t_{2}\right) \simeq y_{e}$. Since $y\left(t_{2}\right) \simeq y_{e}$, this implies that $x\left(t_{2}\right) \simeq y\left(t_{2}\right)$. Which is a contradiction with $\left\|x\left(t_{2}\right)-y\left(t_{2}\right)\right\|$ being appreciable.

CASE $2\left(t_{1,2}=t_{2}-t_{1}\right.$ is limited). By the continuity of the function $\|x(t)-y(t)\|$, there exists at least $t \in\left(t_{1}, t_{2}\right)$ such that

$$
\|x(t)-y(t)\|=\frac{\kappa}{2} .
$$

Let $t_{3}$ and $t_{4}$, respectively, be the first and the last instants in time such that equality (4.29) holds. We have $t_{1}<t_{3} \leq t_{4}<t_{2}$. It is clear that

$$
\|x(t)-y(t)\|<\frac{\kappa}{2}, \quad \forall t \in\left[t_{1}, t_{3}\right) .
$$

It is also clear that

$$
0<\frac{\kappa}{2} \leq\|x(t)-y(t)\| \leq \frac{2}{3} \kappa, \quad \forall t \in\left[t_{4}, t_{2}\right] .
$$


Redefine in (2.2) the initial time $t_{1}=t_{0}$. Let $z\left(\cdot ; t_{1}, x\right)$ denote the solution of (2.2) such that $z\left(t ; t_{1}, x\right)=x(t)$ for $t \in\left[t_{1}-r, t_{1}\right]$. By (4.26) we have

$$
z\left(t ; t_{1}, x\right)=x(t) \simeq y(t) \simeq y_{e}, \quad \text { for } t \in\left[t_{1}-r, t_{1}\right] .
$$

Since $t_{4}-t_{1}$ is limited, according to Theorems 3.5 and Remark 3.6

$$
z\left(t ; t_{1}, x\right) \simeq x(t), \quad \text { on }\left[t_{1}, t_{4}+L\right]=\left[t_{1}, t_{1}+\left(\left(t_{4}-t_{1}\right)+L\right)\right], \quad \forall \text { limited } L>0 .
$$

By Robinson's lemma, there exists $\omega \simeq+\infty$ such that $z\left(t ; t_{1}, x\right) \simeq x(t)$ on $\left[t_{1}, t_{4}+\omega\right]$. Thus we have

$$
z\left(t ; t_{1}, x\right) \simeq x(t), \quad \text { on }\left[t_{1}, t_{5}\right], \text { where } t_{5}=t_{4}+\omega .
$$

By (4.32) and the uniform stability of $y_{e}$ (see Lemma 3.8(1)) we deduce that

$$
z\left(t ; t_{1}, x\right) \simeq y(t)\left(\simeq y_{e}\right), \quad \forall t \geq t_{1} .
$$

Thus, by (4.34) and (4.35)

$$
x(t) \simeq y(t), \quad \forall t \in\left[t_{1}, t_{5}\right] .
$$

Therefore $t_{5}<t_{2}$ since $\left\|x\left(t_{2}\right)-y\left(t_{2}\right)\right\|$ is appreciable.

Take $t=t_{5}$. By (4.36) we have $x\left(t_{5}\right) \simeq y\left(t_{5}\right)$. This contradicts (4.31) since $t_{5} \in$ $\left[t_{4}, t_{2}\right]$.

\section{REFERENCES}

[1] N. N. Bogoliubov and Y. A. Mitropolsky, Asymptotic Methods in the Theory of Non-Linear Oscillations, Hindustan Publishing, Delhi, Gordon and Breach Science Publishers, New York, 1961. MR 25 \#5242. Zbl 151.12201.

[2] F. Diener and M. Diener (eds.), Nonstandard Analysis in Practice, Springer-Verlag, Berlin, 1995. MR 97g:03065. Zbl 848.26015.

[3] F. Diener and G. Reeb, Analyse Non Standard, Collection Enseignement des Sciences, vol. 40, Hermann, Paris, 1989. MR 91k:03157. Zbl 682.26010.

[4] M. Diener and C. Lobry (eds.), Analyse Non Standard et Representation du Réèl. Actes de l'école d'été, [Nonstandard Analysis and Representation of the Reals], Office des Publications Universitaires, 1985. MR 90j:03116. Zbl 678.26010.

[5] M. Diener and G. Wallet (eds.), Mathématiques Finitaires et Analyse Non Standard, vol. 31, no. 1-2, Publications Mathématiques de l'Université de Paris 7, 1989.

[6] A. Halanay, On the method of averaging for differential equations with retarded argument, J. Math. Anal. Appl. 14 (1966), 70-76. MR 33 \#1562.

[7] J. K. Hale, Averaging methods for differential equations with retarded arguments and a small parameter, J. Differ. Equations 2 (1966), 57-73. MR 32 \#6009. Zbl 151.10302.

[8] J. K. Hale and S. M. Verduyn Lunel, Averaging in infinite dimensions, J. Integral Equations Appl. 2 (1990), no. 4, 463-494. MR 92d:34128. Zbl 755.45012.

[9] P. Lochak and C. Meunier, Multiphase Averaging for Classical Systems, Applied Mathematical Sciences, vol. 72, Springer-Verlag, New York, 1988. MR 89k:58254. Zbl 668.34044.

[10] R. Lutz and M. Goze, Nonstandard Analysis. A Practical Guide with Applications, Lecture Notes in Mathematics, vol. 881, Springer-Verlag, New York, 1981. MR 83i:03103. Zbl 506.03021. 
[11] E. Nelson, Internal set theory: a new approach to nonstandard analysis, Bull. Amer. Math. Soc. 83 (1977), no. 6, 1165-1198. MR 57 \#9544. Zbl 373.02040.

[12] A. Robinson, Nonstandard Analysis, American Elsevier, New York, 1974.

[13] J. A. Sanders and F. Verhulst, Averaging Methods in Nonlinear Dynamical Systems, Applied Mathematical Sciences, vol. 59, Springer-Verlag, New York, 1985. MR 87d:34065. Zbl 586.34040.

[14] T. Sari, Stroboscopy and averaging, Colloque Trajectorien à la Mémoire de Georges Reeb et Jean-Louis Callot (Strasbourg-Obernai, 1995), Univ. Louis Pasteur, Strasbourg, 1995, pp. 95-124. MR 97c:34091.

[15] _ Nonstandard perturbation theory of differential equations, Analysis and its Applications, ICMS, Edinburgh, 11-17 August, 1996, http://www.math. univ-mulhouse.fr/ geometry/sari/papers.html.

[16] I. van den Berg, Nonstandard Asymptotic Analysis, Lecture Notes in Mathematics, vol. 1249, Springer-Verlag, Berlin, 1987. MR 89g:03097. Zbl 633.41001.

[17] M. Yebdri, Equations différentielles à retard, Master's thesis, Université de Tlemcen, 1989.

MUSTAPHA LAKRIB: DÉPARTEMENT D’INFORMATIQUE, FACULTÉ DES SCIENCES DE L’INGÉNIEUR, UNIVERSITÉ DJILlALI LIABÈS, B.P. 89, 22000 SidI BEL ABbÈS, ALGERIA

E-mail address: m_7akrib@yahoo.fr 


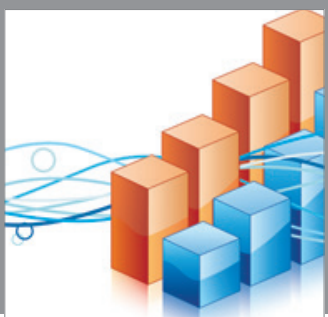

Advances in

Operations Research

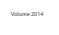

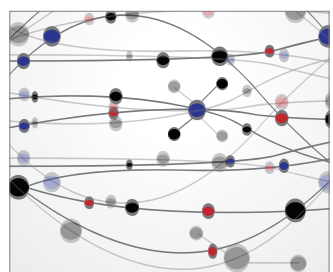

\section{The Scientific} World Journal
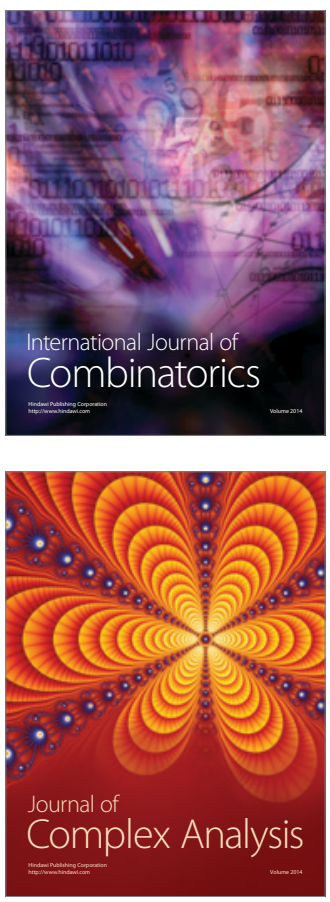

International Journal of

Mathematics and

Mathematical

Sciences
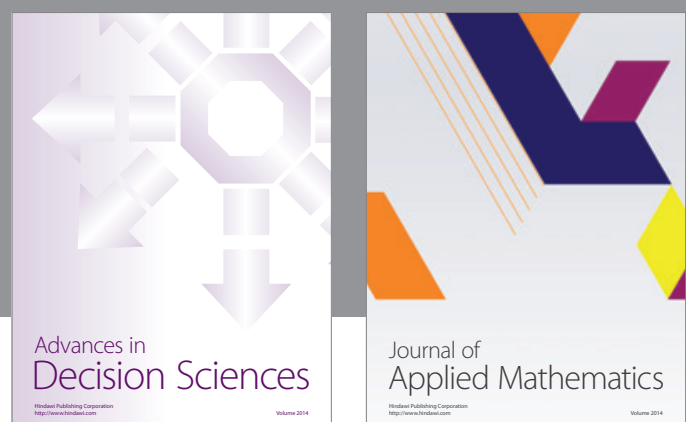

Journal of

Applied Mathematics
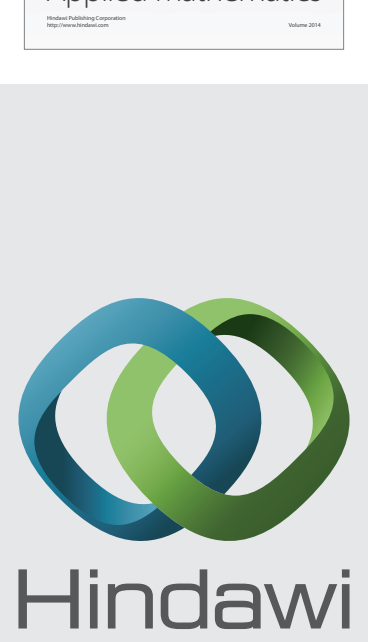

Submit your manuscripts at http://www.hindawi.com
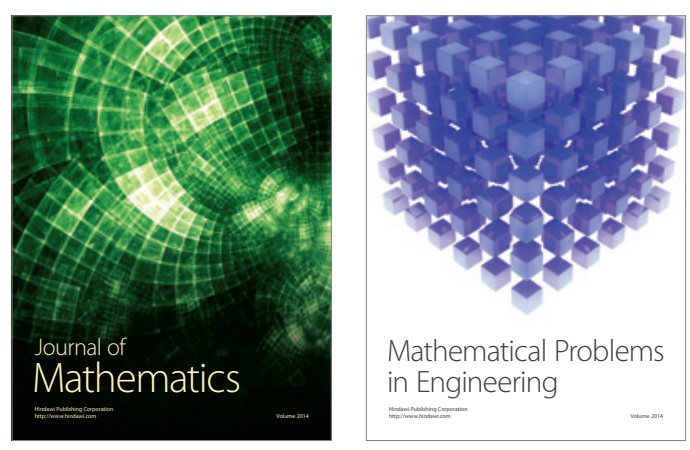

Mathematical Problems in Engineering
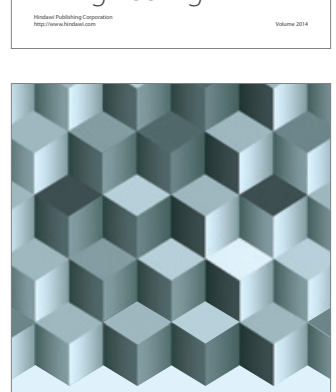

Journal of

Function Spaces
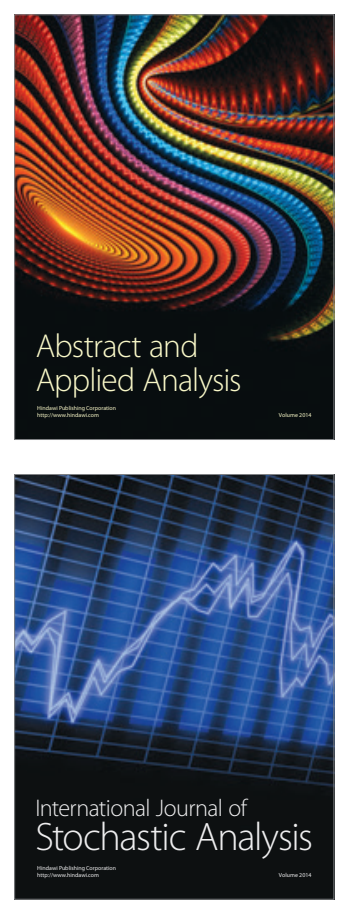

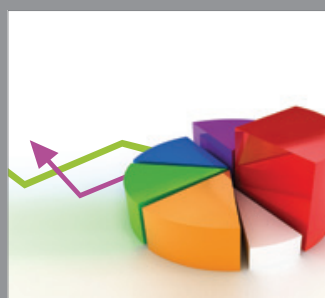

ournal of

Probability and Statistics

Promensencen
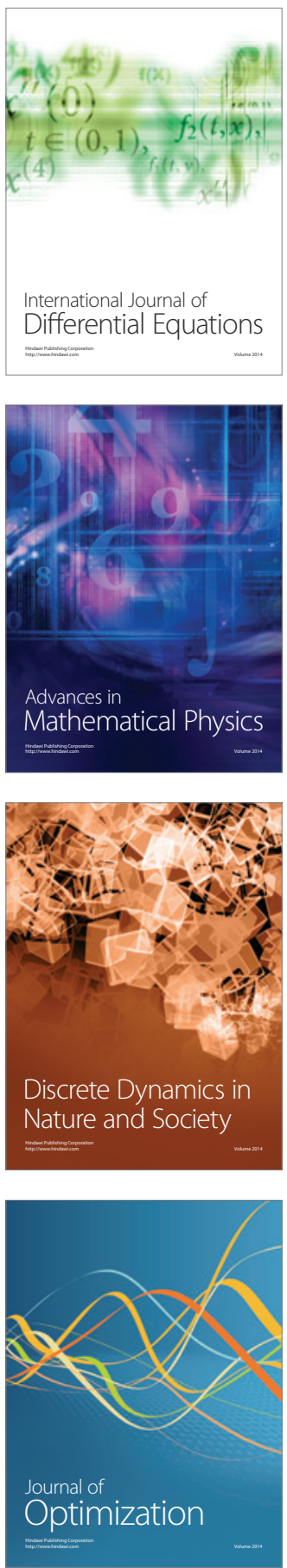